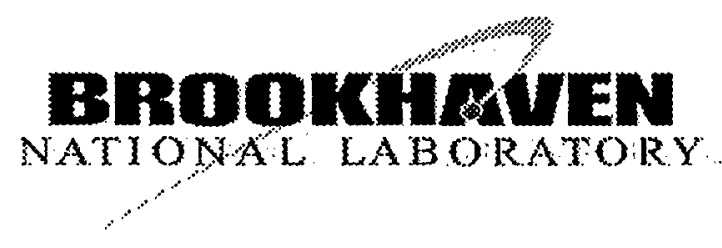

BNL-73352-2004-IR

\title{
Low NOx Burner Development
}

\section{C.R. Krishna and \\ T. Butcher}

September 2004 :

\author{
Energy Science and Technology Department \\ Energy Resources Division \\ Brookhaven National Laboratory \\ P.O. Box 5000 \\ Upton; NY 11973-5000 \\ www:bnl:gov \\ Managed by \\ Brookhaven Science Associates, LLC \\ for the United States Department of Energy under \\ Contract No.'. DE-AC02-98CH10886
}




\section{DISCLAIMER}

This report:was prepared as an account iof work sponsored by an agency of the United States Government. Neither the United States Government nor any: agency thereof, nor any of their employees, nor any of their contractors, subcontractors, or their employees, makes any warranty, express or implied, or assumes any legal liability or responsibility for the accuracy, completeness, or any: third party's use or the results of such use of any information; apparatus; product, or process disclosed, or represents that its use would not infringe privately owned rights. Reference herein to any ispecific commercial product, process, or service by trade name, trademark, manufacturer, or otherwise, does not necessarily constitute or imply its endorsement, recommendation, or favoring by the United States Government or any agency thereof or its contractors or subcontractors. The views and opinions of authors expressed herein do not necessarily state or reflect those of the United States Government or any agency thereof. 
Low NOx burner development ,

\subsection{Objective}

The objective of the task is to develop concepts for ultra low NOx burners. One approach that has been tested previously uses internal recirculation of hot. gases and the objective was to how to implement variable recirculation rates: during burner operation. The second approach was to use fuel oil aerosolization (vaporization) and combustion in a porous medium in a manner similar to gasfired radiant burners.: This task 'is trying the second approach with the use of a somewhat novel, prototype system for aerosolization of the liquid fuel.;

\subsection{Introduction:}

There are a number of approaches to achieving low NOx emissions from a burner and boiler or furnace system. The main contribution to NOx emission is from the thermal NOx formation, which is a strong function of the local ", temperature: Hence, the key to reducing thermal NOx formation is the reduction. or elimination of local zones of high temperature. In the case of liquid fuels, this requires the avoidance of diffusion flames and this has led to the development of blue flame burners. Another approach, which has been primarily used with natural.... gas burners, is the combustion of premixed fuel and air inside or on the surface of porous plates. The rationale is that the combustion occurs at a high intensity. within or at the surface and hence the porous plate is heated to a high temperature.: The solid surface, having a high emissivity, especially compared to an optically thin gas flame, radiates a significant amount of heat to a sink (the heat exchanger) in an appropriately configured system and hence the temperature is lowered below the critical value for NOx formation. Quite low values of NOx: emission have been reported for natural gas burners of this type:

There have been attempts to construct similar burners for using liquid fuels such as kerosene. Clearly, it would not be feasible to spray liquid fuel directly into the porous plates and hence a means must be found to vaporize the fuel and mix it with the necessary air before it can be burned within a porous plate structure. Previous approaches have depended on heating the fuel to a sufficiently high temperature to vaporize it Some of the problems attending this approach are the condensation of the vapors on cold: surfaces, and formation of deposits at vaporizing surfaces (if used) More recently, the so-called cool flame phenomenon has been used to oxidize the fuel partially to produce, presumably, non re-condensable vapors and alleviate the problems noted above.

\subsection{Background:}

Howell et al [1] provide an early review of porous plate burners: There is detailed discussion of the properties of different porous plates; of heat transfer and: flow characteristics and of combustion performance of primarily premixed gaseous fuels (methane) in porous matrix burners. There is a brief discussion of the burning of liquid fuels such as heptane and methanol.. Fuse et al [2] is one attempt by Japanese researchers to develop a porous matrix burner for kerosene. 
They use electric power to vaporize the fuel from an evaporating dish and the vapors are burned in and above the porous matrix. The matrix properties: are chosen so as to increase the radiative heat transfer from the flame to the evaporating fuel and thus minimize the electric power used compared with previous efforts.

Kaplan and Hall [3] burned heptane in a porous ceramic burner The heptane was atomized with a conventional oil burner pressure atomizer at $\sim 100$ psi and premixed with the combustion air Different burner configurations were tried to achieve stable combustion within the porous ceramic plates without flash back and blow-off as well as complete combustion as signified by low carbon monoxide emissions: The configuration changes consisted of adding plates. of porous ceramic and also using different porosity plates among the pieces used to make up the 'burner'. Their experience is particularly interesting as it reflects ours as will be seen below. Their final configuration, which they deemed successful, consisted of a $4 \mathrm{ppcm}$.(pores per centimeter) plate upstream of the burner section and separated from it.'The 'burner', section consisted of a ' flame arrestor'" plate of : $10 \mathrm{ppcm}$ with two plates of $4 \mathrm{ppcm}$ on top of this and finally a $10 \mathrm{ppcm}$ plate as the topmost section: They found that without this top plate of smaller pores, combustion would occur outside the porous plates with blue and/or yellow flames and high $\mathrm{CO}$ emissions. Also, the results were not always repeatable, the reasons for which are not clear, and the stable operating range was quite limited to between equivalence ratios of 0.63 to 0.65 . With the final configuration; the operating range was from 0.57 to 0.67 . The reason given for the success with the fine pore plate on top is that it reduces radiative loss from the top and thus promotes higher temperatures and complete combustion within the rest of the porous plate sections. It should also be noted that their burner section was: insulated with alumina to reduce heat losses. The highest surface combustion: intensity based on their input fuel rate was about $2500 \mathrm{~kW} / \mathrm{m}^{2}$.

Brenner et al. [4] is a recent numerical and experimental investigation of premixed methane and air combustion in a porous inert matrix burner. While this does not treat the problems specific to liquid fuels, some of their results are useful if are trying to burn prevaporized liquid fuel mixed with air. One feature that they emphasize is the use of a preheating region with a porous matrix of fine pores, 18 ppcm in their experiments, upstream of the 'burner plate' with coarser pores, 8 $\mathrm{ppcm}$ in their case. Such an abrupt change in the pore size helps to keep the flame stabilized at the transition region even as the firing rate is modulated, the modulation only changing the length of the 'flame'. The preheater plate's fine : pores are also smaller than the quench diameter and hence the plate doubles as a flame arrestor as long as it does not heat up.: This is ensured by providing for: lateral heat removal by surrounding it with aluminum bars. The porous matrix of large pores is followed by a region of alumina fibers with excellent heat conduction properties. The 'combustion zone'. is insulated to with alumina to minimize heat losses. The surface combustion intensity in these experiments was between $530 \mathrm{~kW} / \mathrm{m}^{2}$ and $2650 \mathrm{~kW} / \mathrm{m}^{2}$.

\subsection{Present Approach}

In the work reported on here, a novel approach to fuel vaporization embodied in a system developed by Vapore, Inc. [http://www.vapore.com] was: 
used to generate the vapor for burning in a porous matrix medium. Its working is described on the company website as follows "The Vapore-Jet?M Capillary Force Vaporizer CFV combines two natural phenomena-capillary force and phase. transition-in an unexpectedly powerful device. By inducing phase transition in a capillary environment, the CFV imparts pressure onto the expanding gas and ejects it with surprising force". The vapor generation rate is controlled by the amount of heat fed to a heating pad that vaporizes the fuel drawn up by a wick. One strong requirement because of the capillary feed is that the pressure at the location of the vapor exit has to be atmospheric. This necessitated the combustion air be drawn into the system by leaving an annular area open above the vapor exit orifice. This complicates efficient mixing of the fuel and vapor and also limits the pressure drop that can be imposed by the porous matrix plates.

The burner system consisted of a quartz tube, with a number of porous matrix plates set one on top of another in the quartz tube. The quartz enabled visual observations and photography. The end of the quartz tube was connected to a metal vent pipe, which housed the fan at a reasonable distance from the burner exit to draw the combustion air. A sampling probe could be introduced ahead of the fan to measure product gas composition.:

\subsection{Experiments and discussions:}

Figure 1 below is a picture of the vaporizing unit with the emerging stream photographed in stroboscopic light to freeze the stream's oscillations. The : observation of the oscillations of the stream, as contrasted with what would be seen in a fuel spray from a conventional atomizer, suggests that it consists of an aerosol of fine drops probably from the condensation of the vapor as it contacts the cold air above the exit orifice: In the next step, this aerosol stream was attempted to be burned on a 'bunsen burner' and a photo of the resulting flame is shown in figure 2 . The flame is yellow as characteristic of an oil flame (home heating oil was used). Following these preliminary tests, it was decided to build the burner set up described above to enable burning the aerosol stream on a porous plate, the main goal of this task. The fuel was switched to kerosene for the rest of the tests for a couple of reasons. It was felt it would be easier to ignite, as we were in uncharted territory and it was not obvious how much the porous plate ${ }_{\mathrm{i}}$ would need to be heated before successful ignition. And, it was felt that there was : more likelihood of the fine pores in the wick of the vaporizing unit clogging with the home heating oil than with kerosene. Figure 3 is a photograph of the set up. 


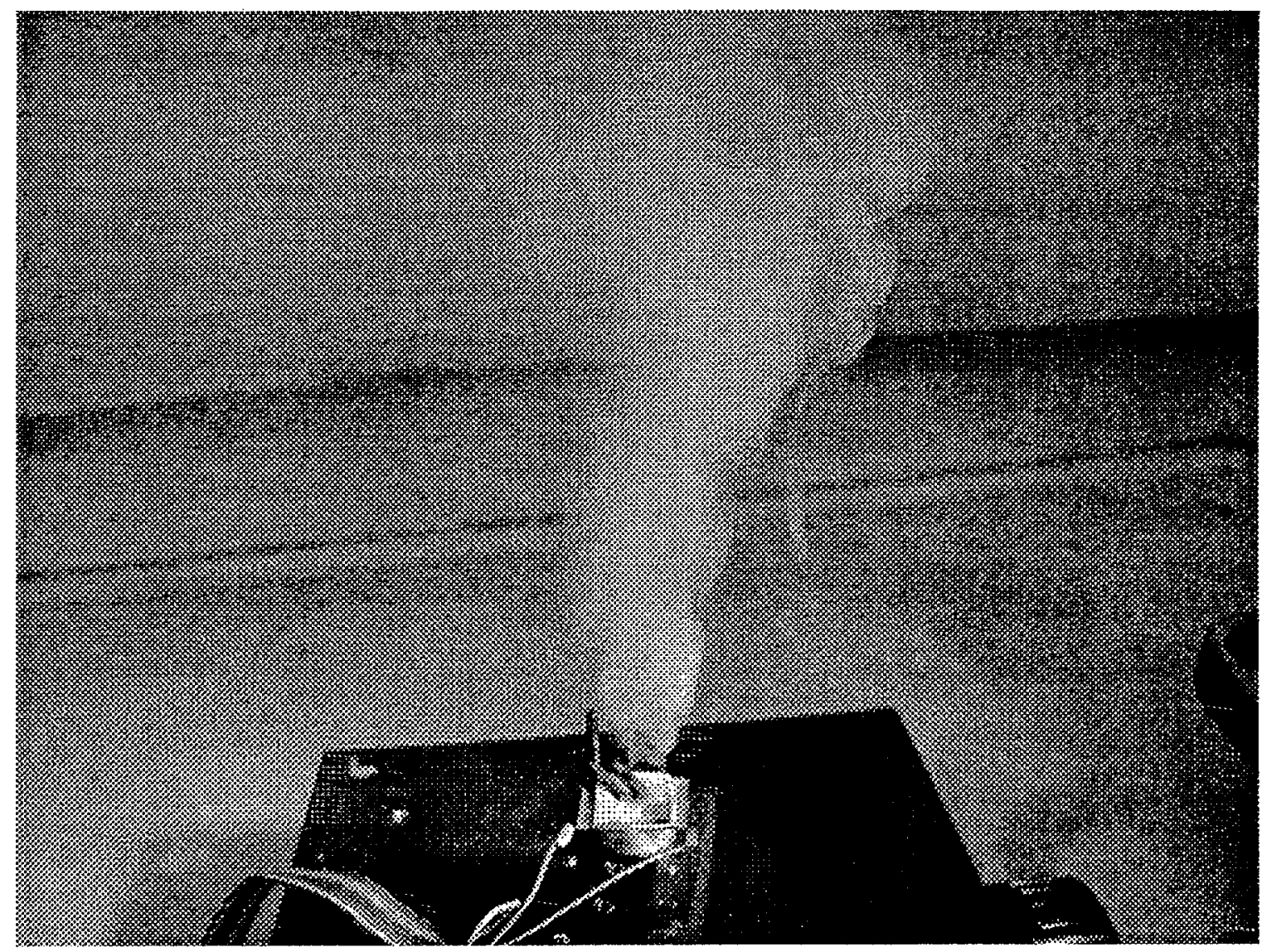

Figure 1. Vapor stream generated by Vapore-Jet unit

Initial experiments used a quartz tube consisting of a length of $7 / 8$ inch diameter: entry tube, just above the Vapore unit; "and into which the aerosol stream was drawn along with combustion air from outside. This ended in a tube of $31 / 4$ inch diameter: (Figure 3 ) This tube had small support lugs for supporting the porous ceramic plates. The initial experiments in this quartz tube were done with one porous ceramic plate of about $1 / 2$ inch thickness and a porosity of 10 pores per inch (ppi) or roughly $4 \mathrm{ppcm}$. The exhaust from the fan was ventilated into the laboratory hood seen behind. The fan speed could be varied to change the air drawn into the system. The "white' cloud seen below the porous plate is presumed. to be condensation of the aerosol: The flame was ignited by a propane torch above the porous plate: A typical 'flame' is shown in figure 4 , in which it can be seen: that a significant part of the burning is taking place above the porous plate This is not quite what is desired: Apart from this, when the plate got hot, there is a tendency for the flame to flash back as well. This suggested that a flame arrester was needed and that a thicker plate for containing the combustion within the plate was also indicated. Typically; a flame arrester would have smaller pore sizes or. more pores per inch so that the passages are less than the 'flame quench': diameter'. The published work reviewed above also suggested that a steep change in pore size would stabilize the flame at the location of this pore size transition. This was implemented by mounting a porous plate of linch thickness with $45 \mathrm{ppi}$ or about $18 \mathrm{ppcm}$ upstream of and abutting the $4 \mathrm{ppcm}$ plate. The tendency to 
flash back was eliminated with this arrangement. However, combustion test results seemed to suggest that complete combustion was not achieved at low enough excess air levels: It was thought that this might be due to low surface combustion intensity and consequently low surface temperatures. Hence; it was. decided to reduce the porous plate size and the quartz tube to fit with it. This arrangement had a similar configuration with a $7 / 8$ inch diameter entry tube opening out to $a$ inch diameter tube in which the porous plate flame arrestor and the porous plate burner plate were set. Figure 5 shows that the flame seems to be stabilized (initiated) at the junction of the flame arrestor and burner plates and to fill the burner plate as well.

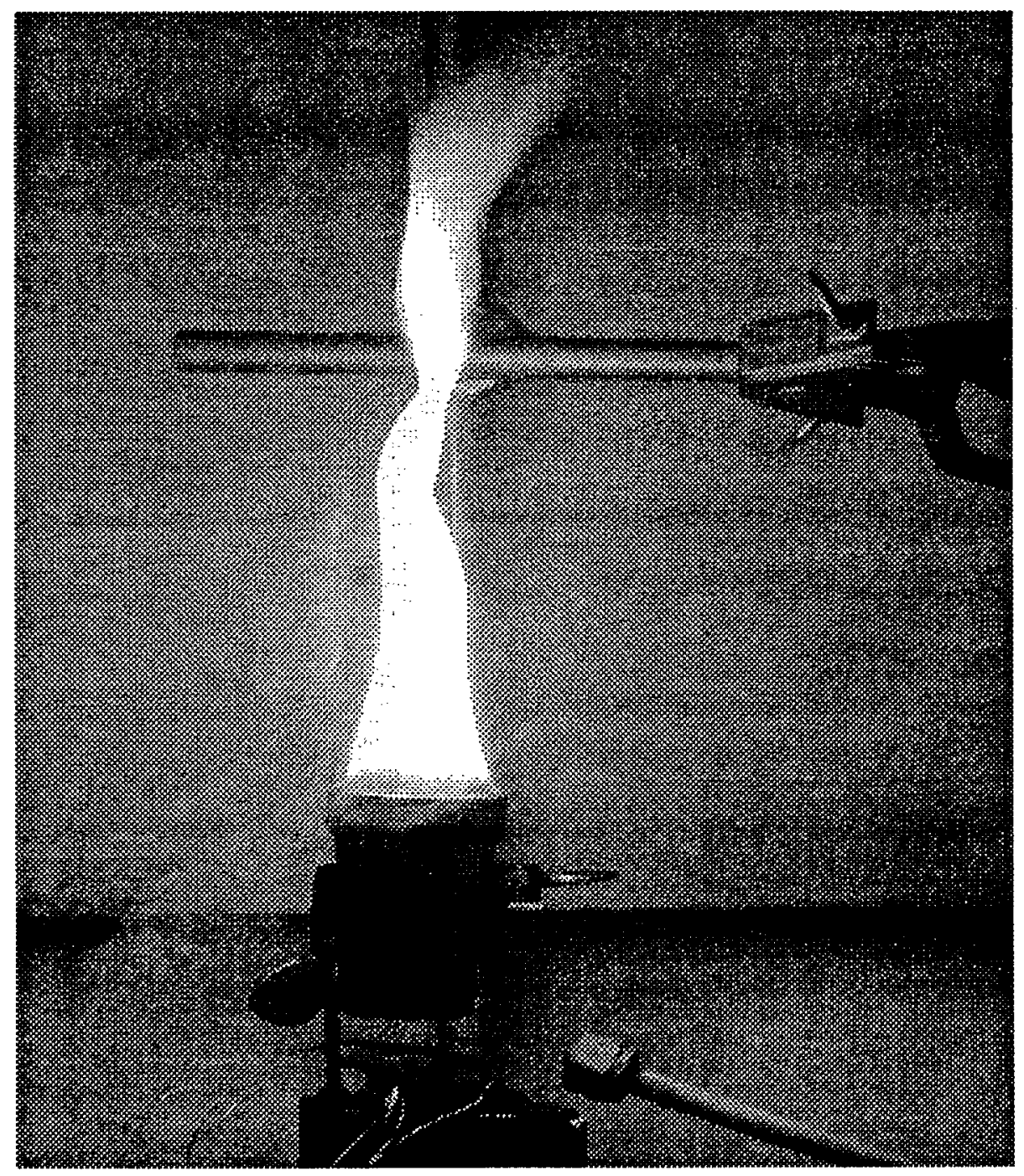

Figure 2. Aerosol stream from Vapore unit burned on a 'Bunsen Burner'. 


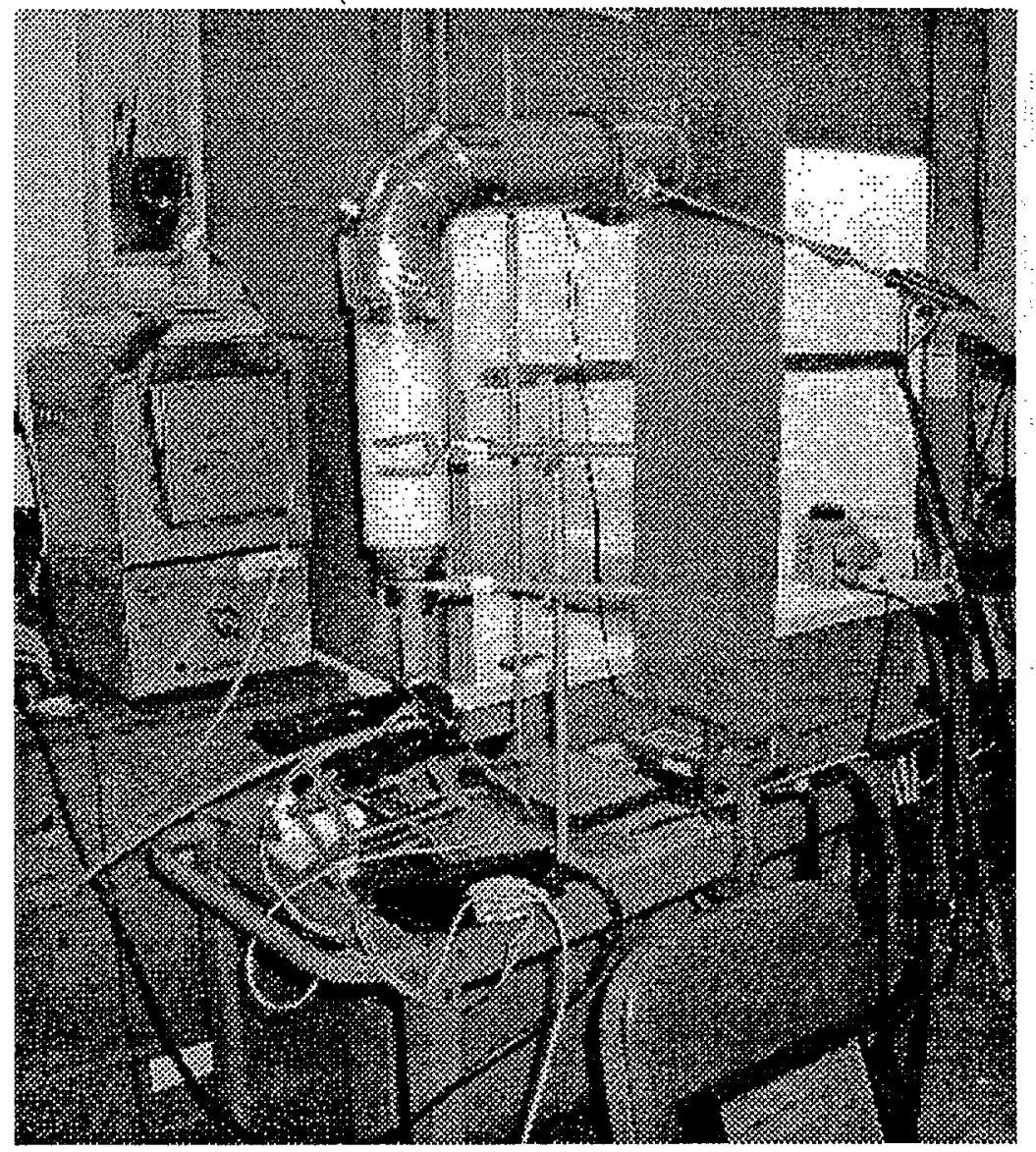

Figure 3. Quartz Burner Set up 


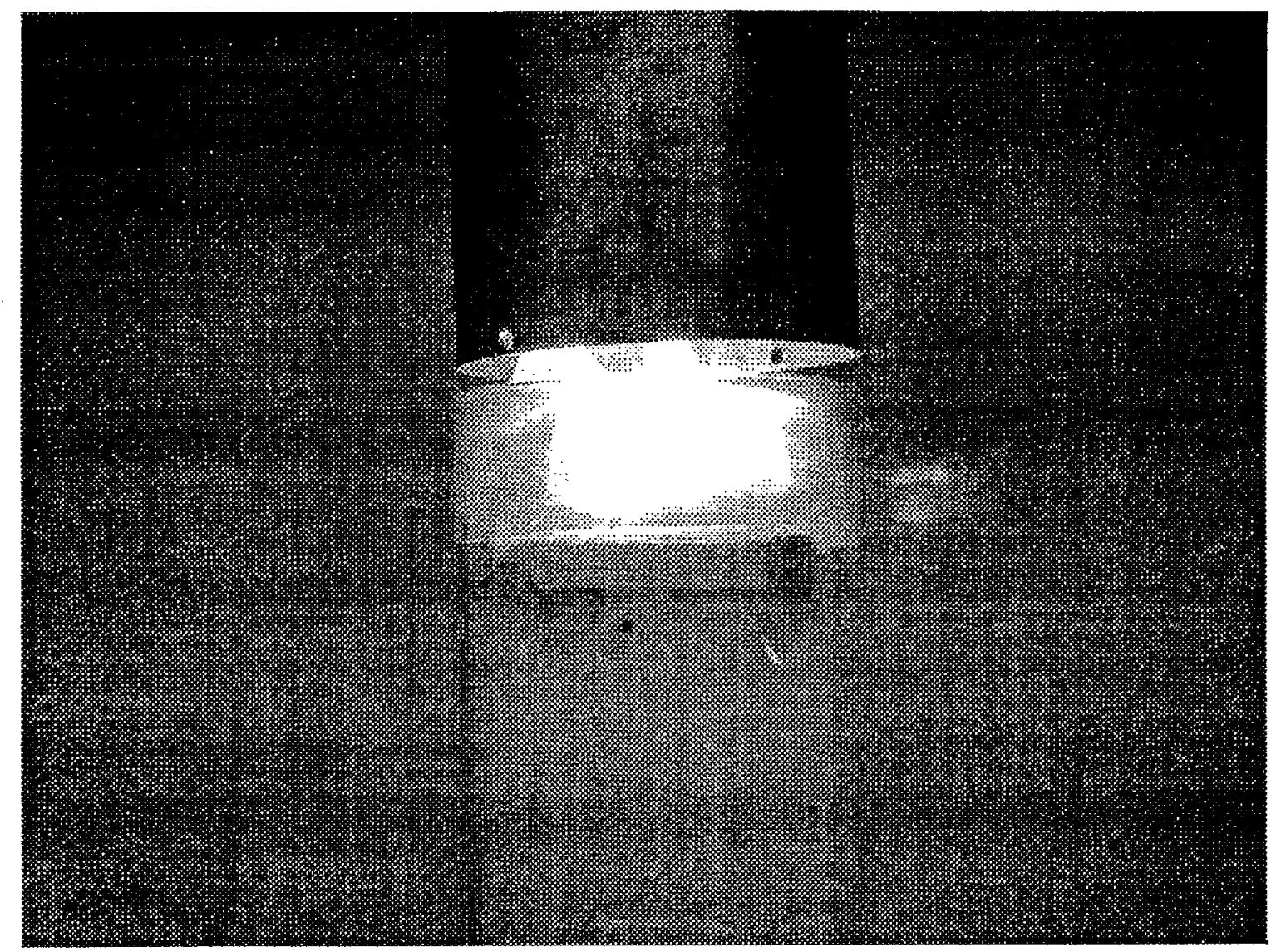

Figure 4. Flame in the initial set up with a single porous plate: 


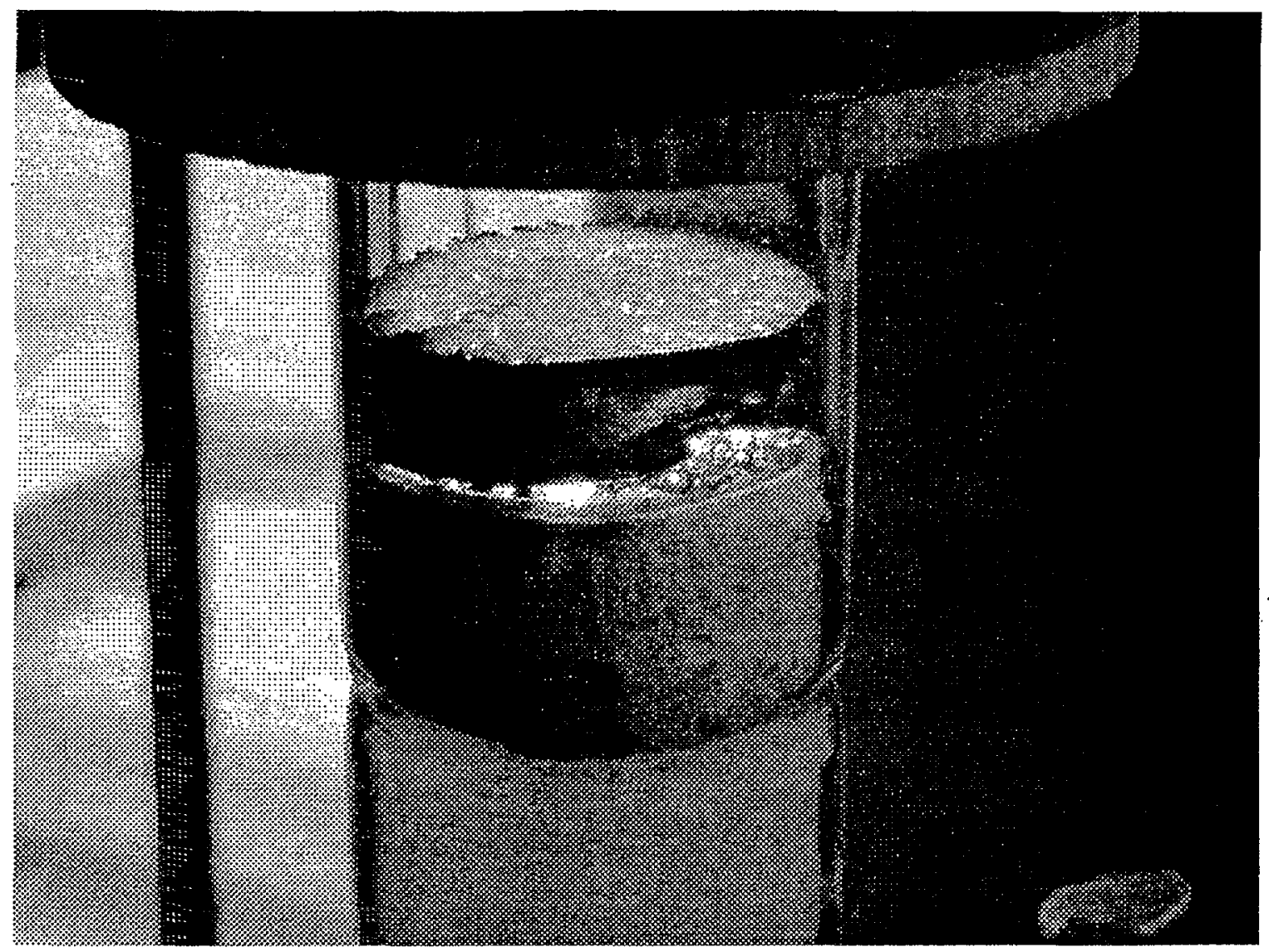

Figure 5. Burning in system with smaller area porous plate

A number of tests were done with this setup at a fuel flow rate of 90 grams per hour: This translates to a surface combustion intensity of about 700 kilowatts per square meter of porous plate area. The lowest excess air achieved was represented by an oxygen concentration of $12.9 \%$ in the combustion product gas corresponding to an excess air of about $230 \%$. The corresponding concentrations for Carbon monoxide and nitrogen oxides were 40 and $14 \mathrm{ppm}$, which translate to $90 \mathrm{ppm}$ and $32 \mathrm{ppm}$ at $3 \%$ oxygen in the product gas. While these values are reasonable, one would like to make improvements to operate at much lower excess air levels and simultaneously reduce the carbon monoxide and nitrogen oxide values. Also, there was some doubt as to whether there were air leaks prior to the measurement location causing oxidation to occur between the porous plate and the sampling probe. Attempts to reduce excess air with the system as set up would cause increases in carbon monoxide and this were not: fruitful. Other improvements, such as heat loss reduction from the porous plate as well as increasing the thickness of the burner plate; seemed to be indicated.

Figure 6 is a photograph of the system with the flame arrestor and two: combustion disks of the same porosity of $\sim 4 \mathrm{ppcm}$. Initial tests in this configuration : indicated somewhat higher $\mathrm{CO}$ emissions and similar NOx emissions as before. The excess air could not be reduced from before : either without large increases in $\mathrm{CO}$. Attempts to reproduce even these results at subsequent dates was unsuccessful The visible combustion zone seems to be localized in the lower part of the first porous plate with insufficient temperature 
and time for complete combustion of the $\mathrm{CO}$. Also, a 'blue' flame is noticed often " above the porous plate suggesting that the $\mathrm{CO}$ is combusted in the freeboard: above. This inconsistency of combustion behavior seems very much similar to that reported by Kaplan and Hall reviewed above and is probably related to acceptable operation over a very narrow range of excess air, which probably is sensitive to heat loss etc. Also, they replaced the second porous disk by one with: more pores per unit length before successful operation. This has not been tried here yet: However, attempts were made to wrap some refractory insulation around, the quartz tube, which did not lead to any improvement.

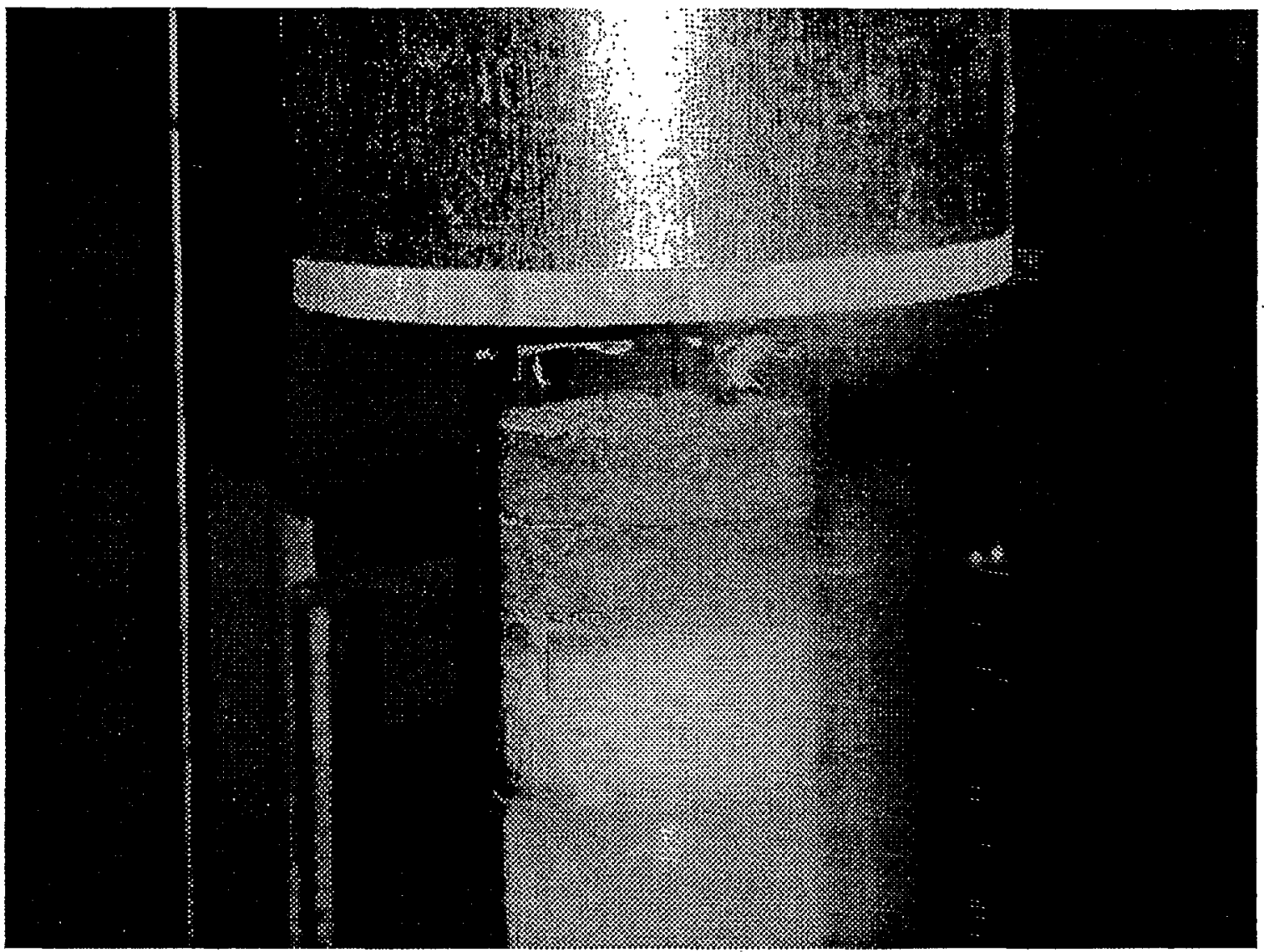

Figure 6. Burner with two combustor disks

\subsection{Recommendations}

The present concept needs improvement before one can decide whether it is viable or not Modifications to the combustion system need to be made to obtain satisfactory combustion at acceptable excess air levels. The modifications 
will include adjustments to the number, thickness and porosity of the porous disks: It will also require insulation of the quartz tube at the appropriate places (combustion zone) and regulation of heat loss from the flame arrestor zone. Combustion intensity: could also need to be adjusted if freeboard burning is to be avoided. Once successful combustion is shown, heat transfer to a heat exchanger should be devised. Low NOx operation should be demonstrated subsequently.

An operational problem in the present configuration is the condensation of: the vapor on the wall of the quartz tube upstream of the flame arrestor disk and drip back of the condensed fuel. In the cumulative tests so far, which have not been over more than half an hour at a stretch, no coking of the fuel in any of the porous disks has been observed. However, long term: operation with diesel fuel without clogging of the capillary:pump and coking in the porous plates should be: demonstrated or means of coping with any problems should $:$ be determined. Similarly, a design to avoid vapor condensation should be developed.

It is recommended that the concept be carried forward till satisfactory combustion and stable operation over a reasonably long time can be shown. 